\title{
Acid-Sensing Ion Channel 1 Is Localized in Brain Regions with High Synaptic Density and Contributes to Fear Conditioning
}

\author{
John A. Wemmie, ${ }^{1,6,7,8}$ Candice C. Askwith, ${ }^{3,7}$ Ejvis Lamani, ${ }^{1}$ Martin D. Cassell, ${ }^{4,6}$ John H. Freeman Jr, ${ }^{5,6}$ and \\ Michael J. Welsh $2,3,6,7$ \\ Departments of ${ }^{1}$ Psychiatry, ${ }^{2}$ Physiology and Biophysics, ${ }^{3}$ Internal Medicine, ${ }^{4}$ Anatomy and Cell Biology, and ${ }^{5}$ Psychology, and ${ }^{6}$ Neuroscience Graduate \\ Program and ${ }^{7}$ Howard Hughes Medical Institute, University of Iowa, Iowa City, Iowa 52242 and ${ }^{8}$ Department of Veterans Affairs Medical Center, Iowa City, \\ Iowa 52242
}

The acid-sensing ion channel, ASIC1, contributes to synaptic plasticity in the hippocampus and to hippocampus-dependent spatial memory. To explore the role of ASIC1 in brain, we examined the distribution of ASIC1 protein. Surprisingly, although ASIC1 was present in the hippocampal circuit, it was much more abundant in several areas outside the hippocampus. ASIC1 was enriched in areas with strong excitatory synaptic input such as the glomerulus of the olfactory bulb, whisker barrel cortex, cingulate cortex, striatum, nucleus accumbens, amygdala, and cerebellar cortex. Because ASIC1 levels were particularly high in the amygdala, we focused further on this area. We found that extracellular acidosis elicited a greater current density in amygdala neurons than hippocampal neurons and that disrupting the ASIC1 gene eliminated $\mathrm{H}^{+}$-evoked currents in the amygdala. We also tested the effect of ASIC1 on amygdala-dependent behavior; ASIC1-null mice displayed deficits in cue and context fear conditioning, yet baseline fear on the elevated plus maze was intact. These studies suggest that ASIC1 is distributed to regions supporting high levels of synaptic plasticity and contributes to the neural mechanisms of fear conditioning.

Key words: ASIC1; localization; CNS; fear conditioning; emotion; learning, memory

\section{Introduction}

Many years ago, it was recognized that rapid acidification of extracellular $\mathrm{pH}$ evokes a transient cation current in central neurons (Gruol et al., 1980; Krishtal and Pidoplichko, 1981). Because brain $\mathrm{pH}$ is tightly regulated in vivo, the physiological significance of this observation has been unclear. However, it was hypothesized that $\mathrm{H}^{+}$-gated currents might be activated during synaptic transmission, because EPSPs acidified the extracellular fluid in hippocampal slices (Krishtal et al., 1987). The discovery of acidsensing ion channels (ASICs), acid-sensing members of the DEG/ ENaC family (Waldmann et al., 1997), suggested several candidate genes and offered an opportunity to explore the physiological role of neuronal $\mathrm{H}^{+}$-evoked currents (Price et al., 2000, 2001; Immke and McCleskey, 2001; Wemmie et al., 2002).

Five ASICs (ASIC1a, 1b, 2a, 2b, and 3; a and b refer to splice

\footnotetext{
Received Feb. 11, 2003; revised April 23, 2003; accepted April 23, 2003.

This work was supported by the Howard Hughes Medical Institute (HHMI) (M.J.W., J.A.W.), a Veteran's Administration Research Career Development Award (J.A.W.), and National Institute of Neurological Disorders and Stroke Grant NS-38890 (J.H.F.). C.C.A. is an Associate and M.J.W. is an Investigator of the HHMI. We thank the University of lowa DNA Core Facility (National Institutes of Health Grant DK-25295) for assistance. We thank Melissa Redeker for excellent assistance, Nicholas Pantazis for helpful discussion, Tom Moninger and the Central Microscopy Research Facility for assistance with microscopy and image analysis, and Christine Bromley and the University of lowa Pathology Research Laboratory for assistance with tissue processing.

Correspondence should be addressed to Dr. John A. Wemmie, Department of Psychiatry, University of lowa, Roy J. and Lucille A. Carver College of Medicine, 500 Eckstein Medical Research Building, lowa City, IA 52242. E-mail: john-wemmie@uiowa.edu.

Copyright $\odot 2003$ Society for Neuroscience $\quad 0270-6474 / 03 / 235496-07 \$ 15.00 / 0$
}

variants) either are activated by acid or modulate acid-gated subunits in heterologous cells (for review, see Welsh et al., 2002; Bianchi and Driscoll, 2002). ASIC subunits assemble into homomultimeric and heteromultimeric channel complexes, and different subunit combinations generate currents with distinct kinetics and $\mathrm{pH}$ sensitivity (Lingueglia et al., 1997; Benson et al., 2002). The properties of $\mathrm{H}^{+}$-gated currents in central neurons generally do not match those of the homomultimeric ASICs, suggesting that heteromultimeric subunit complexes generate the currents (Bolshakov et al., 2002). Because ASIC1a, 2a, and 2b are expressed in brain, combinations of these proteins could be responsible.

Disrupting ASICla in mice eliminated $\mathrm{pH}$ 5-evoked current in hippocampal neurons, identifying it as a key component of $\mathrm{H}^{+}$gated currents (Wemmie et al., 2002). Although ASIC1-null mice were viable, with no obvious anatomic or physiological abnormalities, they exhibited deficits in hippocampus-dependent spatial learning and cerebellum-dependent eyeblink conditioning. Moreover, ASIC1-null mice had impaired long-term potentiation (LTP) at Schaffer collateral-CA1 synapses. They also exhibited reduced excitatory postsynaptic potentials and NMDA receptor activation during high-frequency stimulation. These studies suggested a role for ASIC1 in processes that underlie learning and memory. Consistent with this possibility, in cultured neurons, ASIC1 protein distributed preferentially to dendrites and colocalized with the synaptic marker PSD-95, suggesting that ASIC1 is probably present in the postsynaptic membrane. 
Understanding the physiologic contribution of ASIC1 to brain function requires knowledge of the protein localization. In situ hybridization suggested that ASIC1 mRNA was expressed throughout the CNS, with a greater abundance in hippocampus, cerebral cortex, olfactory bulb, and cerebellum (García-Añoveros et al., 1997; Waldmann et al., 1997). However, mRNA data and subsequent protein localization data (Olson et al., 1998; Alvarez de la Rosa et al., 2003) were limited. Therefore, we used immunohistochemistry to examine ASIC1 protein distribution further. ASIC1 levels differed between brain regions and were high in areas supporting rich synaptic connectivity. One location in which ASIC1 was particularly abundant was the amygdala. Because amygdala-dependent learning has been closely linked to LTP, the amygdala may serve as an especially useful model system for memory (McKernan and Shinnick-Gallagher, 1997) (Rogan et al., 1997). Therefore, we also explored the effect of ASIC1 disruption on $\mathrm{H}^{+}$-evoked currents in amygdala neurons and amygdala-dependent behavior.

\section{Materials and Methods}

Antibody. Polyclonal antiserum (MTY19) was raised in rabbits against the 22 amino acid peptide from the $\mathrm{C}$ terminus of ASIC1, MTYAANILPHHPARGTFEDFTC, coupled to keyhole limpet hemocyanin (Poccono, Canadensis, PA). The IgG fraction was purified using the EconoPac serum IgG purification kit (Bio-Rad, Richmond, CA). Next, an Affi-gel 15 Gel (Bio-Rad) coupled to the nonspecific peptide GTCNAVTMDSDF was used to adsorb additional nonspecificity for $1 \mathrm{hr}$ at $4^{\circ} \mathrm{C}$ (Labquake shaker; Labindustries, Berkeley, CA). To adsorb additional nonspecific components of the sera, we used protein extract obtained from ASIC1 knock-out brains coupled to Affi-gel 15 (Bio-Rad), although this step later proved unnecessary. The eluate from these columns was then bound for $4 \mathrm{hr}$ at $4^{\circ} \mathrm{C}$ to the immunogenic peptide crosslinked to Affi-gel 15. The specific antibody was eluted with $50 \mathrm{~mm}$ glycine/ $\mathrm{HCl}$ at a $\mathrm{pH}$ of $2.5,150 \mathrm{~mm} \mathrm{NaCl}$, neutralized with $1 \mathrm{M}$ Tris at a $\mathrm{pH}$ of 10.4 , and adjusted to $1 \% \mathrm{BSA}, 0.2 \% \mathrm{NaN}_{3}$ for storage at $4^{\circ} \mathrm{C}$.

Immunohistochemistry. Coronal brain slices $(7.5 \mu \mathrm{m})$ were cut on a cryostat (CM 1900; Leica Bannockburn, IL) from tissue that was freshfrozen on dry ice and embedded in Tissue Freezing Medium (Electron Microscopy Sciences, Fort Washington, PA). The slices were dried overnight and hydrated with PBS. They were then fixed in PBS with $4 \%$ formaldehyde, $4 \%$ sucrose for $15 \mathrm{~min}$, followed by $0.25 \%$ Triton X-100 in PBS for $5 \mathrm{~min}$ at room temperature. After two rinses with PBS, endogenous peroxidase activity was quenched with $3 \% \mathrm{H}_{2} \mathrm{O}_{2}$ for 30 min. This was followed with three 5 min washes with PBS and blocking with Tris/ $\mathrm{NaCl} /$ blocking reagent buffer (TNB) (TSA Fluorescence Systems, PerkinElmer Life Sciences, Boston, MA) for 30 min. Purified MTY19 (1:50 in TNB) or anti-calbindin D-28K (Chemicon International, Temecula, CA) was added and allowed to incubate for $2 \mathrm{hr}$. After three 5 min washes with PBS, $\alpha$-rabbit IgG-horse radish peroxidase (HRP) (Amersham Biosciences, Piscataway, NJ) was used as a secondary antibody at 1:200 for $1 \mathrm{hr}$ at $37^{\circ} \mathrm{C}$. After another three 5 min washes with PBS, the signal was amplified by incubating in tyramide solution (TSA Fluorescence Systems, Perkin-Elmer Life Sciences, Boston, MA) for $10 \mathrm{~min}$ at room temperature. Finally, the slices were washed three more times for 5 min with PBS, mounted with Vectashield (Vector Laboratories, Burlingame, CA), and visualized by Bio-Rad MRC 1024 confocal microscope, or Olympus BX-51 epifluorescence microscope (Melville, NY) equipped with Spot RT Slater (Diagnostic Instruments, Sterling Heights, MI). The specific ASIC1 immunostaining was lost in paraffin-embedded tissue and when sections were prepared from brain perfused with formalin in vivo before sectioning.

Immunoblotting. From $500 \mu \mathrm{m}$ Vibratome cut slices (Pelco, Redding, $\mathrm{CA})$, the amygdala, CA1, CA3, posterior cingulate, posterior association cortex, habenula, and thalamus were dissected according to regions surrounded by a dashed line in Figure 1. Tissue homogenate was also obtained from the whole brain and cerebellum. The tissue was homogenized in PBS with protease inhibitors (aprotinin $40 \mu \mathrm{g} / \mathrm{ml}$, leupeptin 40 $\mu \mathrm{g} / \mathrm{ml}$, pepstatin A $20 \mu \mathrm{g} / \mathrm{ml}$, PMSF $40 \mu \mathrm{g} / \mathrm{ml}$, and EDTA $2 \mathrm{~mm}$ ) using a $1 \mathrm{ml}$ Dounce homogenizer (Wheaton, Millville, NJ). The homogenate was cleared of large unground particles with a 10 min centrifugation at 3500 rpm (5415C; Eppendorf Hamburg, Germany). Membrane proteins were precipitated at 70,000 rpm for $30 \mathrm{~min}$ (TL-100; Beckman, Fullerton, CA). The pellet was resuspended in PBS with protease inhibitors. All steps in sample preparation were performed on ice or at $4^{\circ} \mathrm{C}$. Protein concentration was determined (Lowry and Passanneau, 1972), and 100 $\mu \mathrm{g}$ was run on $8 \%$ acrylamide gel and Western blotted. The blot was first probed with MTY19 serum at 1:15,000, followed by $\alpha$-rabbit IgG-HRP (Amersham Biosciences) at 1:10,000. The signal was detected by enhanced chemiluminescence (Pierce, Rockford, IL).

Whole-cell voltage-clamp experiments. Mouse hippocampal cultures were generated from postnatal day 1-2 pups as described previously (Wemmie et al., 2002). Amygdala cultures were generated by the same method except that the amygdala was dissected from $1 \mathrm{~mm}$ coronal sections using the external capsule as a landmark to define the borders of the lateral and basolateral amygdala. Culture medium contained insulin, transferrin, and sodium selenite (I-1884, Sigma, St. Louis, MO), resuspended in $50 \mathrm{ml} \mathrm{H}{ }_{2} \mathrm{O}, 2.5 \mu \mathrm{l} / \mathrm{ml}$ of medium. Whole-cell patch-clamp recordings were performed on neurons from at least two different preparations that were cultured for 1-2 weeks. Electrodes (3-5 M $\Omega$ ) were filled with intracellular solutions containing (in mM): $120 \mathrm{KCl}, 10 \mathrm{NaCl}$, $2 \mathrm{MgCl}_{2}, 5$ EGTA, 10 HEPES, and $2 \mathrm{ATP}$. The $\mathrm{pH}$ was adjusted to 7.2 with KOH. Extracellular solutions contained (in $\mathrm{mM}$ ): $128 \mathrm{NaCl}, 2 \mathrm{CaCl}_{2}, 1$ $\mathrm{MgCl}_{2}, 5.4 \mathrm{KCl}, 5.55$ glucose, 10 HEPES, and 10 MES. To inhibit spontaneous activity, $0.5 \mu \mathrm{M}$ tetrodotoxin, $5 \mu \mathrm{M}$ CNQX, $15 \mu \mathrm{M}$ bicuculline methiodide, and $25 \mu \mathrm{M}$ DL-2-amino-5-phosphonovaleric acid were added to the extracellular solutions. The $\mathrm{pH}$ was adjusted with tetramethylammonium hydroxide (TMA-OH) and the osmolarity adjusted with TMA-Cl. Neurons were held at $-80 \mathrm{mV}$ for recording, and extracellular $\mathrm{pH}$ was 7.4 unless otherwise indicated. All chemicals were obtained from Sigma.

Elevated-plus maze. A maze was constructed from stainless steel with a Plexiglas base ( 36 inches tall) and two pair of arms $(2 \times 115 / 8$ inches $)$ intersecting at right angles. One pair of arms was closed and had six inch walls on three sides. The two open arms lacked walls. A $2 \times 2$ inch intersection connected the four arms. Naive mice $(+/+, n=11 ;-/-$, $n=11$ ) were placed onto the center of the maze and allowed $5 \mathrm{~min}$ to roam freely. Activity was recorded by a video camera suspended above the maze. A trained technician blinded to genotype recorded the time each animal spent in the closed arms, open arms, and stationary in the corner of the closed arms. The number of entries into the open central intersection was also determined. Statistical significance was tested with a two-sample $t$ test.

Auditory fear conditioning. On day 1 , naive mice $(+/+, n=7 ;-/-$, $n=9$ ) were placed in a conditioning chamber (Lafayette Instrument, Lafayette, IN). After $3 \mathrm{~min}$, they were presented with a tone $(80 \mathrm{~dB}, 20$ $\mathrm{sec})$ that coterminated with an electric foot-shock $(1 \mathrm{~mA}, 1 \mathrm{sec})$. A total of seven pairings of the tone and shock were delivered, separated by $1 \mathrm{~min}$ intervals. Mice were then returned to their home cage. On day 2, to minimize freezing to context, the lights were dimmed, burgundy poster board was used to change the color of the back wall and ceiling, a wire mesh floor grate was inserted, white bench paper was placed under the floor grid, and the paper was dabbed with 1 drop of peppermint extract. The animals were placed in the conditioning chamber, observed for 3 $\mathrm{min}$, and then presented with the same tone continuously for $6 \mathrm{~min}$, minus the foot-shock. Freezing (defined as a crouched posture and an absence of movement) during 1 min intervals was quantified from videotapes by a trained observer blinded to genotype. Three $-/-$ mice and one $+1+$ mouse were excluded from the training data because they climbed onto the wall of the chamber during at least one interval. Although this did not interfere with the conditioning protocol, it did interfere with scoring and disqualified them from the ANOVA with repeated measures. One $+/+$ mouse was excluded from the study because its tail was inadvertently pinched as it was being placed into the chamber. Another $+/+$ mouse was excluded because its freezing response was $>3 \mathrm{SD}$ from the mean. The context fear conditioning protocol was similar, except on day 1 , the mice received three shocks and no tone was presented 
$(+/+, n=8 ;-/-, n=7)$. On day 2 , the same chamber was used without changing the context.

\section{Results and Discussion}

ASIC1 immunolocalization in the brain Previous in situ hybridization studies suggested that ASIC1 transcripts were abundant in layers CA1 through CA4 of the hippocampus (García-Añoveros et al., 1997; Waldmann et al., 1997). In addition, we have shown previously that disrupting ASIC1 impairs Schaffer collateral-CA1 LTP and adversely affects spatial learning (Wemmie et al., 2002). Therefore, we asked where in the hippocampal circuit ASIC1 protein was located. An affinitypurified rabbit polyclonal antibody against the C-terminal 22 amino acids of mouse ASIC1 was used to immunolabel coronal sections of mouse brain (Fig. 1A). As a control, we used ASIC1 -/- brains. In the hippocampus, the hilus (polymorphic layer) of the dentate gyrus showed the most prominent ASIC1 staining. This region is occupied by inhibitory and excitatory interneurons as well as mossy fibers and CA3 dendrites (Fig. $1 B$ ).

In contrast, ASIC1 immunostaining in CA1 and CA2 was relatively weak (Fig. $1 A, C)$. Others have suggested that epitope masking may obscure ASIC1 detection in the brain (Olson et al., 1998). To address this possibility, we also immunoblotted protein obtained from the dentate gyrus and CA1. Although ASIC1 was detected, it was dramatically reduced in CA1 compared with the dentate gyrus (Fig. 1D). Thus, although ASIC1 may have important effects on CA1 function (Wemmie et al., 2002), the amount of protein in this region may be sparse relative to other areas.

Because ASIC1 distribution in the hippocampus was different than anticipated, we asked about its distribution elsewhere in the brain. Previous studies reported that ASIC1 mRNA was elevated in the cerebral cortex (García-Añoveros et al., 1997; Waldmann et al., 1997). Consistent with those reports, we found abundant ASIC1 protein in a number of specific cortical regions (Figs. 1A, 2, 3). ASIC1 staining was evident in the anterior and posterior cingulate cortex (Figs. $1 A, 2 A, B$ ). The sensory and motor cortices were also immunopositive (Figs. 1A, 3). A subdomain of the sensory cortex in which ASIC1 staining was prominent was the whisker barrel field (Figs. 1 A, 2C), an area that has served as a valuable model system for analyzing cortical plasticity (for review, see Fox, 2002). In contrast, ASIC1 immunostaining was low in the ectorhinal, perirhinal, and piriform cortex (Figs. 1 A, 3).

ASIC1 immunostaining in sensorimotor and cingulate cortex
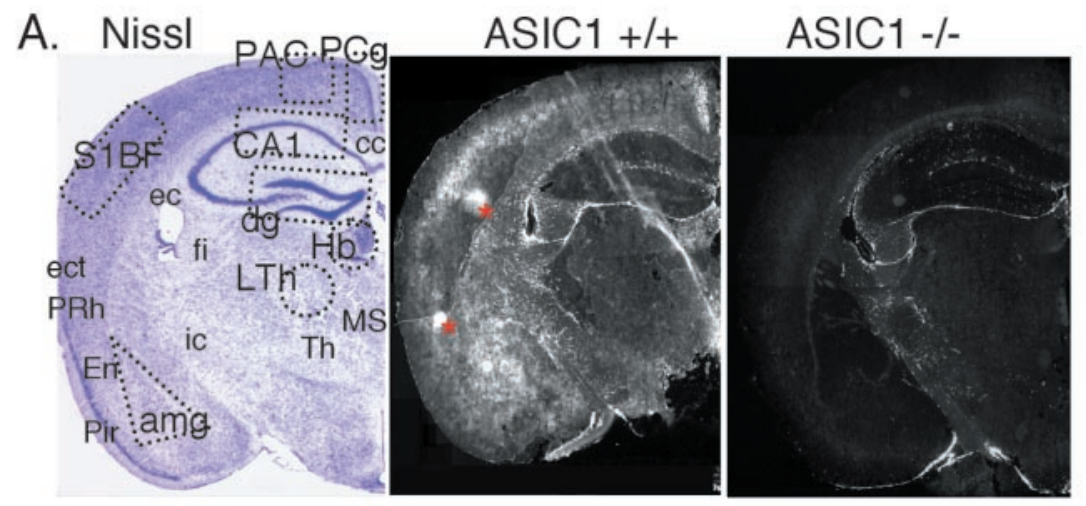

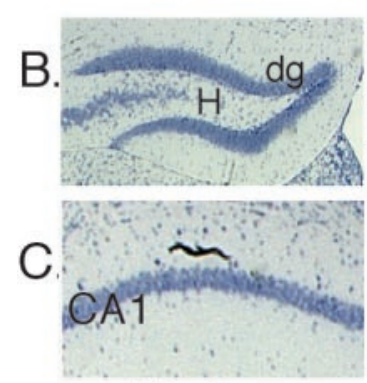

Niss!
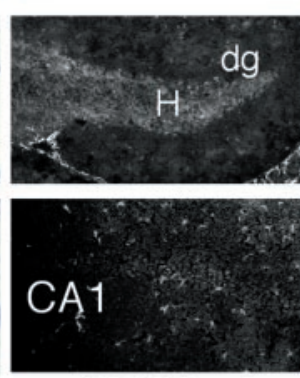

ASIC1 +/+

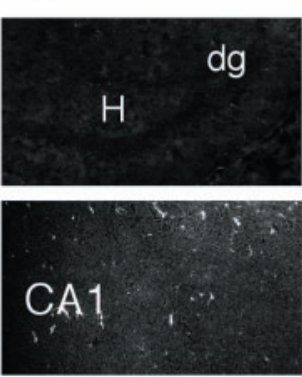

ASIC1 -/-
Figure 1. ASIC1 immunolocalization in forebrain. A, Coronal sections were stained for Nissl substance or immunolabeled for ASIC1 protein in $+1+$ and $-/-$ mice. Areas marked by dashed lines in the Nissl-stained section are the areas dissected to prepare protein extracts for Western blotting in $D$ and Figure $6 B$. Asterisks in ASIC1+/+ hemisphere denote areas of nonspecific staining that did not occur bilaterally or in multiple sections. $B, C$, Enlarged images of dentate gyrus and CA1 respectively. D, Western blot of ASIC1 protein in $100 \mu \mathrm{g}$ protein extract from dentate gyrus and CA1. amg, amygdala; cc, corpus callosum; dg, dentate gyrus; ec, external capsule; ect, ectorhinal cortex; En, endopiriform nuclei; fi, fimbria; $\mathrm{Hb}$, habenula; $\mathrm{H}$, hilus (polymorphic layer); ic, internal capsule; LTh, lateral thalamus; MS, medial septal nuclei; PAC, parietal association cortex; Pir, piriform cortex; PCg, posterior cingulate cortex; PRh, perirhinal cortex; S1BF, somatosensory barrel field; Th, thalamus.

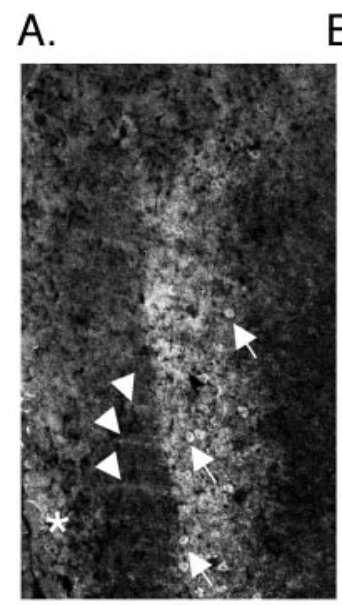

post. cingulate

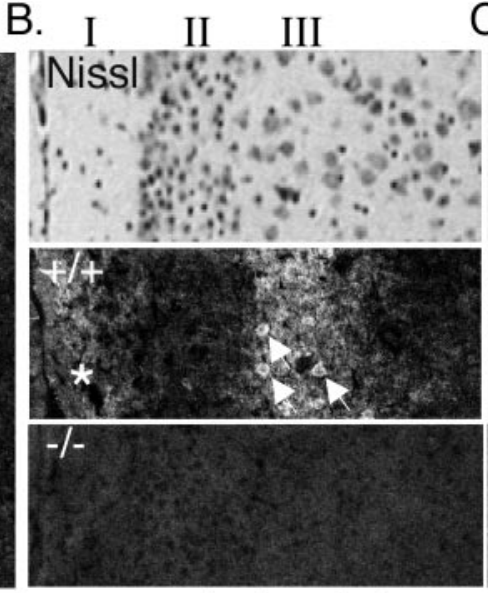

post. cingulate

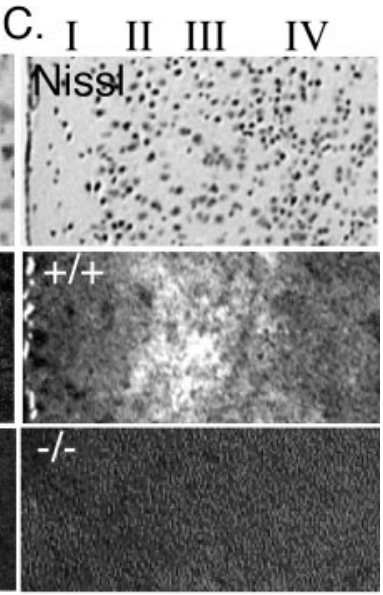

barrel cortex
Figure 2. ASIC1 immunolocalization in cortex. $A, B$, Immunolabeling in the posterior (post.) cingulate cortex. Stripes extending through layer Il are labeled with an arrowhead. Positive-staining pyramidal cells in layer III are labeled with arrows. *ASIC1-specific staining in layer I. C, ASIC1 immunostaining is also elevated in layer III of barrel cortex.

tended to be elevated in layer III. For example, in the posterior cingulate, immunolabeling could be seen on pyramidal cell bodies in layer III (Fig. $2 A, B$, arrows) and also in layer I near the brain surface (Fig. $2 A$, asterisk). We also consistently observed stripes of staining perpendicular to the cortical layers and extending between layers I and III, possibly caused by apical dendrites extending from pyramidal neurons in the deeper layers (Fig. 2A, 

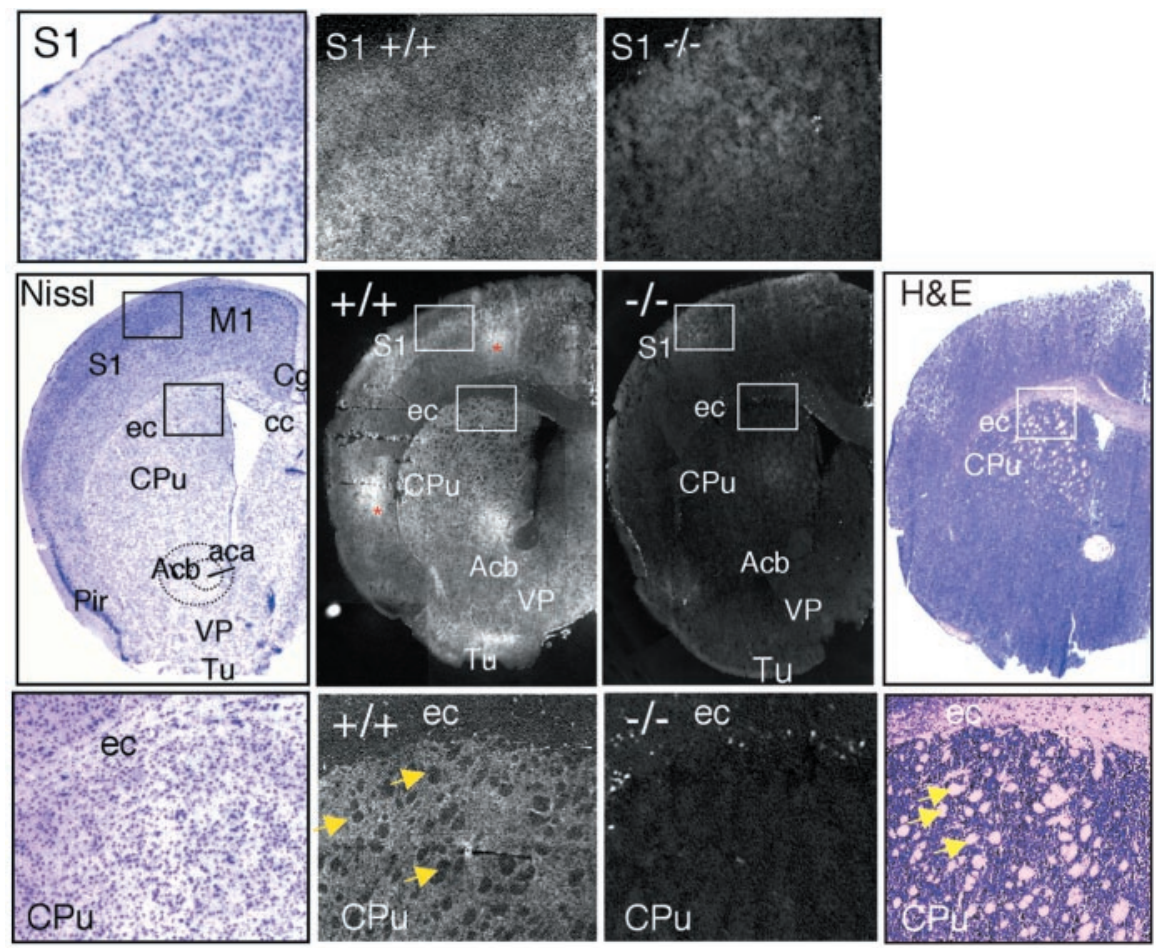

Figure 3. Immunolocalization of ASIC1 in the sensorimotor cortex and striatum. Coronal sections through the forebrain were stained for Nissl substance, hematoxylin and eosin (H\&E), or ASIC1 protein in ASIC1 + / + or - / - mice. Center row, staining of representative coronal slices. Top row, insets of somatosensory cortex at higher magnification. Bottom row, insets of external capsule/corpus callosum and striatum at higher magnification. White matter tracts are labeled with arrows. ASIC1 immunolabeling was noticeably reduced in the white matter tracts. Areas of staining that were not present bilaterally and not present in multiple slices, suggesting nonspecific staining, are marked with an asterisk. aca, anterior commissure; Acb, accumbens nucleus; cc, corpus callosum; $\mathrm{Cg}$, cingulate cortex; $\mathrm{CPu}$, caudate/putamen (striatum); ec, external capsule; M1, primary motor cortex; Pir, piriform cortex; S1, somatosensory cortex; VP, ventral pallidum; Tu, olfactory tubercle.

arrowhead). ASIC1 staining in barrel and motor cortex was also preferentially distributed to layer III (Figs. 1A, 2C). The significance of layer III specificity is not clear, although it is interesting to note that an NMDA receptor-dependent form of LTP in this layer has been implicated in barrel cortex function (Fox, 2002).

In addition to the cortex, we observed strong ASIC1 staining in certain subcortical structures, including the basal ganglia (Fig. 3). ASIC1 labeling was readily apparent in the striatum, in which it was distributed in gray matter, and was slightly more abundant dorsally and laterally (Fig. 3), regions that preferentially receive sensorimotor cortical input. The strong signal in gray matter of the striatum contrasted sharply with weak white matter staining, giving the ASIC1 distribution a dappled appearance (Fig. 3). We also observed strong ASIC1 in the ventral pallidum, olfactory tubercle, and nucleus accumbens (Fig. 3). The basal ganglia serve an important role in voluntary movement. The striatum and nucleus accumbens may also contribute to motivation and appetitive behavior and have been linked to addiction in humans (for review, see Cardinal et al., 2002; Hyman and Malenka, 2001). Yet, ASIC1 knock-out mice performed normally on the accelerating Rotarod (Wemmie et al., 2002) and displayed normal activity on the elevated plus maze (see below). Nevertheless, the high level of ASIC1 in the striatum suggests that given the appropriate challenge, ASIC1-null mice might exhibit abnormal striatumdependent behavior.

In contrast to the basal ganglia, ASIC1 immunostaining in the thalamus was rather weak, with the exception of the habenula and the medial septal nuclei (Fig. $1 A$ ). The significance of the selective distribution between subcortical structures is not yet clear.
We also tested for ASIC1 protein in the olfactory bulb, because ASIC1 mRNA was reported to be elevated there (Waldmann et al., 1997). We found ASIC1 protein localized preferentially to the glomerular layer and most evident within glomeruli (Fig. 4, arrows). Immunolabeling of periglomerular cells was less intense, causing the striking glomerular pattern to stand out (Fig. 4). Glomeruli provide a site for synaptic contact between olfactory sensory neurons and intrinsic olfactory bulb neurons, including periglomerular cells, mitral cells, and tufted cells. Because olfactory sensory neurons are continuously replaced throughout life, synapses in the glomerulus undergo constant remodeling (for review, see Shepherd and Greer, 1998). This high degree of plasticity is unique in the mammalian brain. The strong ASIC1 signal in the glomeruli is consistent with the ability of ASIC1 to affect synaptic function (Wemmie et al., 2002).

The cerebellum contains abundant ASIC1 mRNA (García-Añoveros et al., 1997; Waldmann et al., 1997), and our eyeblink conditioning studies suggested that ASIC1 may have important effects on cerebellum-dependent learning (Wemmie et al., 2002). In the cerebellum, ASIC1 staining was particularly strong in the molecular layer, and in both the molecular and granule cell layers, it was distributed diffusely, suggesting that its source is rather widespread (Fig. 5). In these layers, the most prevalent cell types are granule and Purkinje cells. Because both produce $\mathrm{H}^{+}$evoked currents (Allen and Attwell, 2002; Escoubas et al., 2000; C. Askwith, unpublished observations), both probably contribute to the strong ASIC1 labeling.

The ASIC1 staining in the granule layer suggested that it may be distributed to granule cell dendrites, which are located there and receive afferent mossy fiber input. ASIC1 may also be present in granule cell axons, which project into the molecular layer where ASIC1 staining was strong (Fig. 5). However, because Purkinje cells are known to express large $\mathrm{H}^{+}$-gated currents, Purkinje cell dendrites may account for much of the ASIC1 protein in the molecular layer. A Purkinje cell-specific antibody (anticalbindin D-28K) produced a similar diffuse pattern in the molecular layer (Fig. 5C). Purkinje cell axons traverse the white matter to form presynaptic terminals in the deep nuclei; however, ASIC1 staining in these areas was not greater than that in the - /controls. The absence of detectable ASIC1 protein in Purkinje cell axons suggests that in these cells, it may be preferentially localized to dendrites.

A general pattern that emerged in the study of ASIC1 localization in brain was a tendency for it to be enriched in areas receiving strong excitatory corticofugal input (cortical projections); examples include the cortex, striatum, nucleus accumbens, and dentate gyrus of the hippocampus. These structures are interconnected in a circuit referred to as the limbic corticostriatal loop (for review, see Cardinal et al., 2002). Components of this circuit are thought to contribute to the emotional importance of exter- 


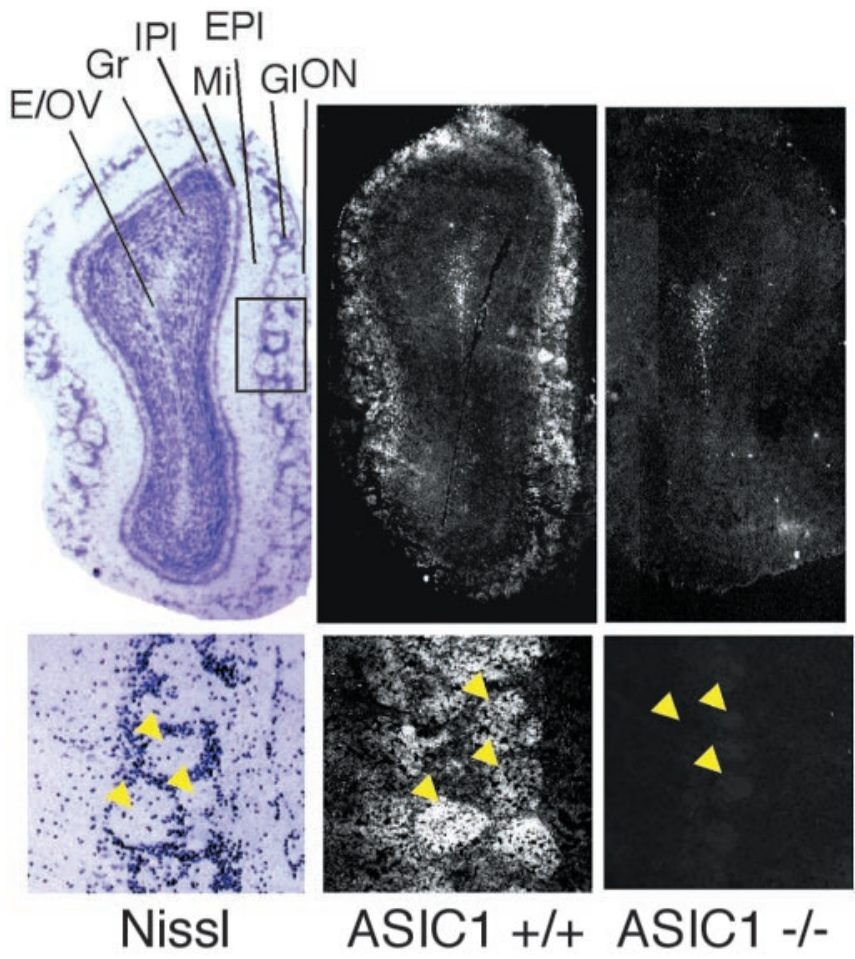

Figure 4. Immunolocalization of ASIC1 in the olfactory bulb. Coronal sections through the olfactory bulb were stained for Nissl substance or immunolabeled for ASIC1 protein in ASIC1 $+/+$ and $-/-$ mice. Higher magnifications at bottom demonstrate ASIC1 immunostaining in glomeruli (arrowheads). E/OV, ependymal and subendymal layer/olfactory ventricle; EPI, external plexiform layer; GI, glomerular layer; Gr, granule cell layer; IPI, internal plexiform layer; $\mathrm{Mi}$, mitral cell layer; $\mathrm{ON}$, olfactory nerve layer.

nal stimuli and/or their expression. Another important component of this circuit is the amygdala complex, in which ASIC1 immunolabeling was intense, particularly in the lateral and basolateral nuclei (Figs. 1A, 6A). We obtained a similar result using Western blot to compare ASIC1 protein levels. ASIC1 was especially abundant in the amygdala and was present at higher levels than in the hippocampus or thalamus, for example (Fig. 6B).

These data are in contrast to those described recently by Alvarez de la Rosa et al. (2003), which suggested that ASIC1 protein was broadly distributed in neurons throughout the brain without a trend toward a particular brain region or cellular domain. One advantage of our experiments is that we used ASIC1 knock-out mice as a control for specificity. Moreover, multiple approaches, including immunohistochemistry, Western blotting, and measurement of $\mathrm{H}^{+}$-gated current density (see below), all suggested that ASIC1 protein is preferentially distributed to specific domains. These studies are also consistent with our earlier experiments in cultured neurons transfected with ASIC1, which showed a dendritic and synaptic pattern of ASIC1 localization (Wemmie et al., 2002).

\section{ASIC1 is a required component of $\mathrm{H}^{+}$-activated channels in the amygdala}

To explore the electrophysiological impact of ASIC1 expression in the amygdala, we measured $\mathrm{H}^{+}$-gated currents in cultured amygdala neurons. Reducing extracellular $\mathrm{pH}$ to 5.0 evoked large transient currents in the majority of ASIC1 +/+ neurons (93\%; $n=27$ ) (Fig. 7). In contrast, none of the amygdala neurons from ASIC1 - /- mice generated transient currents in response to $\mathrm{pH}$ $5(n=29)$. These data indicate that ASIC1 makes a critical con-

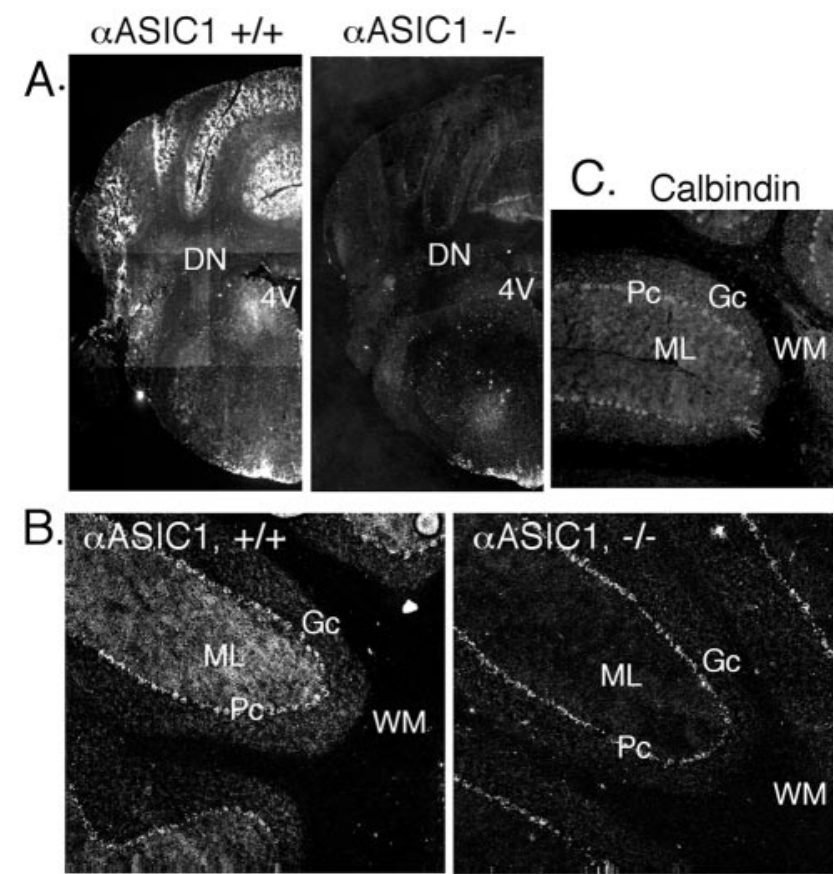

Figure 5. Immunolocalization of ASIC1 in the cerebellum. ASIC1 immunohistochemistry in coronal $(A)$ and parasagittal $(B)$ sections of the cerebellum. $C$, Immunostaining with anticalbindin D-28K antibody in fresh-frozen tissue. $4 \mathrm{~V}$, fourth ventricle; $\mathrm{DN}$, deep cerebellar nuclei; $\mathrm{Gc}$, granule cell layer; ML, molecular layer; Pc, pyramidal cell layer; WM, white matter.

\section{A \\ B.}
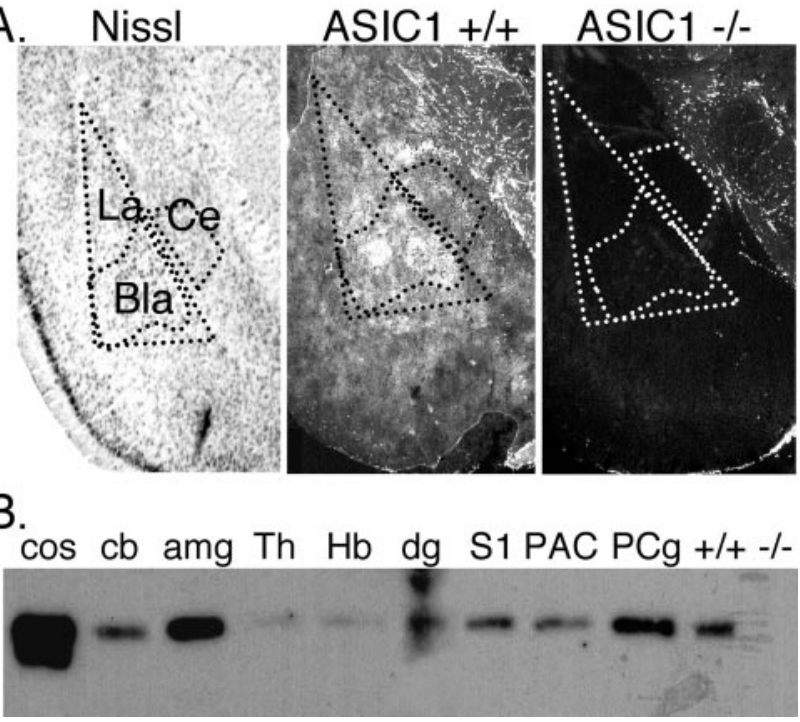

Figure 6. $\quad A, A S I C 1$ immunolocalization in the amygdala complex. Bla, basolateral nucleus; Ce, central nucleus; La, lateral nucleus. B, Western blotting of ASIC 1 protein in $100 \mu \mathrm{g}$ of protein extract per lane isolated from indicated brain region. Cos-7 cells transfected with mASIC1, cos. Because the entire cerebellum was used to generate the cb extract, the subcortical structures with little ASIC1 may have diluted out the high expression level seen by immunohistological staining in the cerebellar cortex (Fig. 5). $+/+$ and $-/-$, whole-brain extract from ASIC1 $+/+$ or $-/-$ mouse; amg, amygdala; cb, cerebellum; dg, dentate gyrus; $\mathrm{Hb}$, habenula; $\mathrm{SI}$, somatosensory barrel field; Th, thalamus; PAC, parietal association cortex; PCg, posterior cingulate cortex.

tribution to $\mathrm{H}^{+}$-gated current in these cells. We also found that the mean current density of $\mathrm{H}^{+}$-gated currents was more than threefold greater in amygdala than in hippocampal neurons (Fig. 7). Thus, compared with hippocampus, the amount of ASIC1 

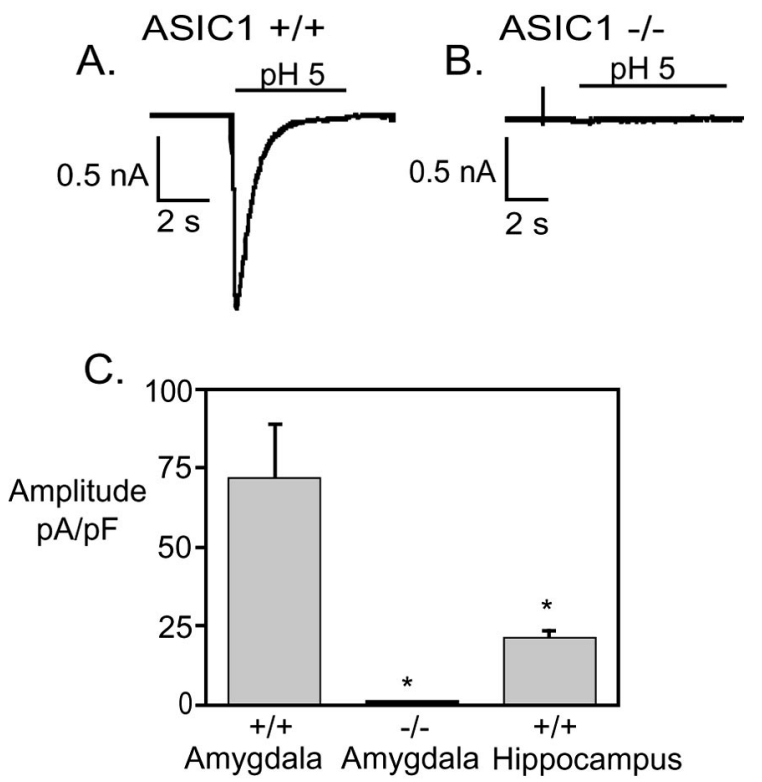

Figure 7. Proton-gated currents in amygdala neurons. $A, B$, Representative recordings of $\mathrm{pH}$ 5 evoked response in amygdala neurons from $\mathrm{ASIC} 1+1+$ and $-1-$ mice. $C$, Average current density of peak pH 5 -evoked response in amygdala neurons from ASIC1 $+/+(n=14)$ and $-/-(n=18)$ mice and hippocampal neurons from ASIC1 $+/+$ mice $\left(n=67 ;{ }^{*} p<0.01\right)$.

protein and the average number of functional ASIC channels are much greater in the amygdala.

\section{ASIC1 and amygdala-dependent behavior}

Finding that ASIC1 protein was present in a number of structures in the limbic corticostriatal loop and that ASIC1 protein and $\mathrm{H}^{+}$-gated currents were abundant in the amygdala suggested that ASIC1 might play an important role in behaviors controlled by these structures (Cardinal et al., 2002). To test this hypothesis, we examined the effect of ASIC1 disruption on performance in the elevated plus maze, a test of baseline fear. Both ASIC1 $+/+$ and $-/-$ mice spent the majority of time in the closed arms $(+/+=198 \pm 4 \mathrm{sec} ;-/-=217 \pm 3 \mathrm{sec}$; mean \pm SEM; $p=$ 0.21 ), suggesting that the two groups found the open arms similarly aversive. In addition, the number of open arm entries $(+/+$ $=12 \pm 1 ;-/-=12 \pm 1$; mean $\pm \mathrm{SEM} ; p=0.96$ ), motor activity (time motionless in the corner of the closed arms, $+/+=78 \pm 5$ sec; $-/-=75 \pm 5$ sec; mean \pm SEM; $p=0.88)$, and risk assessment (time scanning edge, $+/+=16 \pm 0.5 \mathrm{sec} ;-/-=14 \pm 0.7$ sec; mean \pm SEM; $p=0.56$ ) was similar for the two genotypes. Together, these data suggest that activity and baseline fear are normal in ASIC1 $-/-$ mice.

The amygdala is a key component of the circuitry for learned fear (Faneslow and LeDoux, 1999). Our previous finding that ASIC1 disruption impaired synaptic plasticity and memory (Wemmie et al., 2002) raised the possibility that loss of ASIC1 might alter amygdala-dependent learning. We tested cued fear conditioning by repeatedly presenting a tone and foot shock and measuring the percentage of time spent freezing during 1-min intervals. With repeated stimuli, both the $+/+$ and $-/-$ mice froze more, although the $-/-$ mice lagged slightly behind. However, the robust freezing of $-I-$ mice in the final minute of training (Fig. $8 A$ ) suggested that $-/-$ animals were capable of expressing a strong fear response when trained extensively. The next day, we tested the ability of the tone to induce freezing in the absence of a shock. A continuous tone was presented for $6 \mathrm{~min}$.
A.

B.
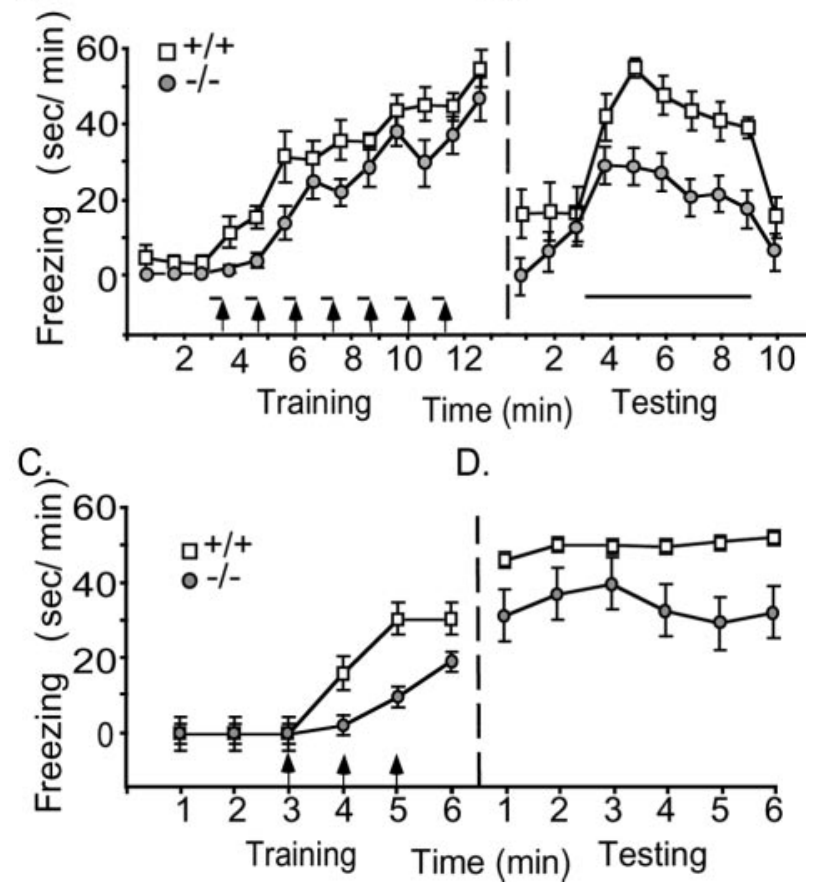

Figure 8. Behavioral analysis of learned fear. $A, B$, Cued fear conditioning. The amount of freezing in 1 min intervals was determined during training $(A)$ and testing $(B)$. During testing, the ASIC1 $-/-$ mice froze significantly less than $+/+$ controls with the presentation of the conditioned stimulus (intervals $4-9)(p=0.02)(+/+, n=5 ;-1-, n=9)$. C, D, Context fear conditioning. The difference in freezing between $+/+$ and $-1-$ mice was significant during training (intervals $4-6 ; p=0.002)$ and during testing $(p=0.03)(+/+, n=7$; $-/-, n=8)$. Foot shock, arrows; tone, bars. Statistical significance was tested by ANOVA with repeated measures.

Animals of both genotypes responded with an increase in freezing, indicating the occurrence of auditory fear conditioning (Fig. $8 B)$. However, the ASIC1-null mice spent significantly less time freezing than their wild-type littermates.

The presence of ASIC1 in the primary sensory cortex and sensory neurons raised the possibility that the $-/-$ mice performed poorly because of a sensory deficit. However, after each shock without exception, both $-I-$ and $+/+$ mice responded by jumping, vocalizing, or running. The average duration of the response $(+/+, 1.7 \pm 0.2 \mathrm{sec} ;-/-, 1.5 \pm 0.2 \mathrm{sec}$; mean $\pm \mathrm{SD}$; $p=0.057)$, and the percentage of shocks eliciting a vocalization $(+/+, 80.7 \pm 26.4 \% ;-/-, 91.6 \pm 23.7 \% ; p>0.2)$ was similar between the two groups. These results agree with our previous studies, which found that unconditioned responses to electrical shock during eyeblink conditioning were normal in ASIC1 - /mice (Wemmie et al., 2002). In addition, at the behavioral level, we have found no differences in $+/+$ and $-/-$ animals in mechanosensation, thermal sensation, or allodynia to skin or muscle stimulation (data not shown). Finally, both genotypes performed similarly on an accelerating Rotarod (Wemmie et al., 2002). Together, these data suggest that the observed differences in fear conditioning were not likely to have been the result of a sensory or motor deficit.

To test whether the fear conditioning deficit was restricted to cue, the mice were also conditioned to context. Again, the -/mice acquired the freezing response more slowly on day 1 (Fig. $8 C$ ) and froze less on day 2, suggesting that the problem in fear conditioning is not restricted to auditory stimuli. 


\section{Conclusions}

Consistent with the previously suggested role for ASIC1 in synaptic function (Wemmie et al., 2002), the ASIC1 protein was preferentially distributed to brain regions with strong excitatory synaptic input. Because ASIC1 was abundant in the lateral and basolateral nuclei of the amygdala, it is possible that the freezing deficit in the ASIC1-null mice is because of impaired learning, especially because baseline fear on the elevated plus maze was intact. However, ASIC1 was also expressed in other regions of the fear circuit, for example, the cingulate cortex, nucleus accumbens, and central nucleus of the amygdala, structures thought to contribute to the emotional importance of external stimuli and/or the expression of fear (Cardinal et al., 2002). Thus, ASIC1 might affect multiple brain regions underlying the acquisition and expression of the fear response. Additional studies will be necessary to delineate the multiple possible effects of ASIC1 on behavior. Understanding how ASIC1 contributes to brain function offers new possibilities for elucidating the molecular mechanisms of memory and emotion.

\section{References}

Allen NJ, Attwell D (2002) Modulation of ASIC channels in rat cerebellar Purkinje neurons by ischaemia-related signals. J Physiol (Lond) 543:521-529.

Alvarez de la Rosa D, Krueger SR, Kolar A, Shao D, Fitzsimonds RM, Canessa CM (2003) Distribution, subcellular localization and ontogeny of ASIC1 in the mammalian central nervous system. J Physiol (Lond) 546:77-87

Benson CJ, Xie J, Wemmie JA, Price MP, Henss JM, Welsh MJ, Snyder PM (2002) Heteromultimerics of $\mathrm{DEG} / \mathrm{ENaC}$ subunits form $\mathrm{H}^{+}$-gated channels in mouse sensory neurons. Proc Natl Acad Sci USA 99:2338-2343.

Bianchi L, Driscoll M (2002) Protons at the gate: DEG/ENaC ion channels help us feel and remember. Neuron 34:337-340.

Bolshakov KV, Essin KV, Buldakova SL, Dorofeeva NA, Skatchkov SN, Eaton MJ, Tikhonov DB, Magazanik LG (2002) Characterization of acidsensitive ion channels in freshly isolated rat brain neurons. Neuroscience 110:723-730.

Cardinal RN, Parkinson JA, Hall J, Everitt BJ (2002) Emotion and motivation: the role of the amygdala, ventral striatum, and prefrontal cortex. Neurosci Biobehav Rev 26:321-352.

Escoubas P, De Weille JR, Lecoq A, Diochot S, Waldmann R, Champigny G, Moinier D, Menez A, Lazdunski M (2000) Isolation of a tarantula toxin specific for a class of proton-gated $\mathrm{Na}^{+}$channels. J Biol Chem 275:25116-25121.

Faneslow MS, LeDoux JE (1999) Why we think plasticity underlying pavlovian fear conditioning occurs in the basolateral amygdala. Neuron 23:229-232.

Fox K (2002) Anatomical pathways and molecular mechanisms for plasticity in the barrel cortex. Neuroscience 111:799-814.
García-Añoveros J, Derfler B, Neville-Golden J, Hyman BT, Corey DP (1997) $\mathrm{BNaC} 1$ and $\mathrm{BNaC} 2$ constitute a new family of human neuronal sodium channels related to degenerins and epithelial sodium channels. Proc Natl Acad Sci USA 94:1459-1464.

Gruol DL, Barker JL, Huang LY, MacDonald JF, Smith Jr TG (1980) Hydrogen ions have multiple effects on the excitability of cultured mammalian neurons. Brain Res 183:247-252.

Hyman SE, Malenka RC (2001) Addiction and the brain: the neurobiology of compulsion and its persistence. Nat Rev Neurosci 2:695-703.

Immke DC, McCleskey EW (2001) Lactate enhances the acid-sensing $\mathrm{Na}^{+}$ channel on ischemia-sensing neurons. Nat Neurosci 4:869-870.

Krishtal OA, Pidoplichko VI (1981) A "receptor" for protons in small neurons of trigeminal ganglia: possible role in nociception. Neurosci Lett 24:243-246.

Krishtal OA, Osipchuk YV, Shelest TN, Smirnoff SV (1987) Rapid extracellular $\mathrm{pH}$ transients related to synaptic transmission in rat hippocampal slices. Brain Res 436:352-356.

Lingueglia E, de Weille JR, Bassilana F, Heurteaux C, Sakai H, Waldmann R, Lazdunski M (1997) A modulatory subunit of acid sensing ion channels in brain and dorsal root ganglion cells. J Biol Chem 272:29778-29783.

Lowry OH, Passanneau JV (1972) A multistep metabolite assay (for inorganic phosphate). In: A flexible system of enzymatic analysis, Ed 3 (Lowry OH, Passonneau JV, eds), pp 86-92. New York: Academic.

McKernan MG, Shinnick-Gallagher P (1997) Fear conditioning induces a lasting potentiation of synaptic currents in vitro. Nature 390:607-611.

Olson TH, Riedl MS, Vulchanova L, Ortiz-Gonzalez XR, Elde R (1998) An acid sensing ion channel (ASIC) localizes to small primary afferent neurons in rats. Neuron 9:1109-1113.

Price MP, Lewin GB, McIlwrath SL, Cheng C, Xie J, Heppenstall PA, Stucky CL, Mannsfeldt AG, Brennan TJ, Drummond HA, Qiao J, Benson CJ, Tarr DE, Hrstka RF, Yang B, Williamson RA, Welsh MJ (2000) The mammalian sodium channel BNC1 is required for normal touch sensation. Nature 407:1007-1011.

Price MP, McIllwrath SL, Xie J, Cheng C, Qiao J, Tarr DE, Sluka KA, Brennan TJ, Lewin GR, Welsh MJ (2001) The DRASIC cation channel contributes to the detection of cutaneous touch and acid stimuli in mice. Neuron 32:1071-1083.

Rogan MT, Stäubli UV, LeDoux JE (1997) Fear conditioning induces associative long-term potentiation in the amygdala. Nature 390:604-607.

Shepherd GM, Greer CA (1998) Olfactory bulb. In: The synaptic organization of the brain, Ed 4 (Shepherd GM, ed), pp 159-204. New York: Oxford UP.

Waldmann R, Champigny G, Bassilana F, Heurteaux C, Lazdunski M (1997) A proton-gated cation channel involved in acid-sensing. Nature 386:173-177.

Welsh MJ, Price MP, Xie J (2002) Biochemical basis of touch perception: mechanosensory function of degenerin/epithelial $\mathrm{Na}^{+}$channels. J Biol Chem 277:2369-2372.

Wemmie JA, Chen J, Askwith CC, Hruska-Hageman AM, Price MP, Nolan BC, Yoder PG, Lamani E, Hoshi T, Freeman JHJ, Welsh MJ (2002) The acid-activated ion channel ASIC contributes to synaptic plasticity, learning, and memory. Neuron 34:463-477. 